\title{
LETTERS
}

\section{Acute chikungunya virus infection and asthma}

\section{To the Editors:}

From March 2005 to June 2006, Réunion Island, a French overseas territory in the Indian Ocean, faced an explosive chikungunya outbreak (a mosquito-borne disease caused by an alphavirus of the Togaviridae family). It was estimated that about 300,000 people were affected by the disease [1]. Many patients experienced related chikungunya disease symptoms (fever, polyarthralgia, skin rashes, diarrhoea, vomiting). To date, there are no studies describing a respiratory tract involvement due to chikungunya infection. During this period, many patients complained of respiratory symptoms such as de novo dyspnoea or a worsening of various respiratory symptoms in patients with asthma. We hypothesised that acute chikungunya infection (ACI) may induce airway hyperresponsiveness (AHR) and therefore increases symptoms of asthma.

The study was prospectively conducted from December 2005 to May 2006. All patients had ACI defined as symptoms (fever, polyarthralgia) that occurred in the 10 days preceding the evaluation, with a laboratory-confirmed diagnosis of acute chikungunya: positive RT-PCR, positive immunoglobulin (Ig)M serological test. All asthmatic patients had a history of significant reversibility of airflow obstruction. Pulmonary function testing (PFT) was performed with a phletysmograph (Medisoft ${ }^{\circledR}$, Dinant, Belgium) at baseline and after inhaling $400 \mu \mathrm{g}$ of salbutamol. Results (forced expiratory volume in $1 \mathrm{~s}$ (FEV1), forced vital capacity (FVC), maximum mid-expiratory flow at $25-75 \%$ of FVC) were compared with former data recorded with the same phletysmograph. Patients were asked to fill a validated asthma 15-question questionnaire to assess quality of life (mini-Asthma Quality of Life Questionnaire (AQLQ)). The questionnaire generated an overall score and a four-domain score: symptoms, five questions; activities, four questions; emotion, three questions; environmental exposure, three questions. Each ranged 1-7, with higher scores indicating better quality of life in the last 2 weeks. Treatment for asthma was carefully recorded. Nonasthmatics subjects had no history of asthma or any respiratory disease. They did not receive any corticosteroids during the past 3 months. A baseline pulmonary function test was performed, followed by a metacholine challenge test [2]. A provocative concentration causing a $20 \%$ fall in FEV1 baseline value was considered significant. If PFT did not change after inhalation of $16 \mathrm{mg} \cdot \mathrm{mL}^{-1}$ of metacholine (six consecutives tests), the challenge was considered as negative. When the challenge test was found to be positive, the patient was invited to perform a supplemental test 4 6 months after acute chikungunya.

32 (8 males, 24 females) asthmatics ( $44 \pm 19$ yrs of age) were evaluated. The mean duration of asthma was $18 \pm 15$ yrs. Global Initiative for Asthma (GINA) stratification was recorded as follows: intermittent 4 , mild persistent 5 , moderate persistent 18 , severe persistent 5 . Maintenance therapy was not statistically different before and during ACI. PFT values were not statistically different from their baseline best-known values. The mini-AQLQ score was compared for 27 patients. There was no statistical change for the overall score. The four domains of the mini-AQLQ score were analysed. The items related to physical activities tended to be worse during acute chikungunya, but did not reach significance $(p=0.06)$ (table 1$)$. 11 nonasthmatics subjects (6 females, 5 males, $38 \pm 24$ yrs of age) performed the metacholine challenge test. 10 subjects had a negative test. The single patient with a positive test was challenged again 6 months after acute chikungunya but the

TABLE 1 Functional and clinical characteristics of asthmatic patients before and during acute chikungunya infection

Befor

During

p-value

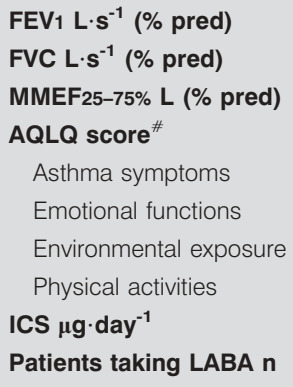

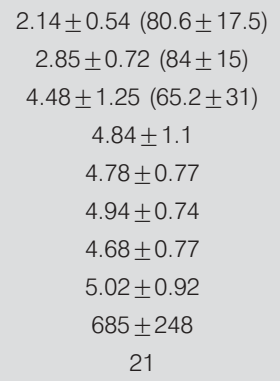

$2.1 \pm 0.5(79.8 \pm 16.7)$
$2.79 \pm 0.81(83.4 \pm 15.6)$
$4.68 \pm 1.3(63.8 \pm 32.4)$
$4.45 \pm 0.92$
$4.96 \pm 1$
$5.12 \pm 1.01$
$4.98 \pm 0.67$
$4.12 \pm 0.98$
$694 \pm 264$

22
0.18

0.38

0.47

0.29

0.83

0.65

0.81

0.06

0.79

1

Data are presented as mean \pm SD unless stated otherwise. FEV1: forced expiratory volume in $1 \mathrm{~s}$; \% pred: \% predicted; FVC: forced vital capacity; MMEF25-75\%: maximum mid-expiratory flow at 25-75\% of FVC; AQLQ: Asthma Quality of Life Questionnaire; ICS: inhaled corticosteroids; LABA: Iong-acting $\beta_{2}$-agonist. PFT and mini-AQLQ results were compared with the paired t-test. Maintenance asthma therapy was compared with Fisher's exact test. ${ }^{\#}$ : Data available for 27 patients. 
test remained positive. This patient had a personal history of atopy and transient wheezing. He was considered to have intermittent asthma as a secondary diagnosis.

This study performed during Réunion Island's chikungunya outbreak provides two interesting findings. First, asthmatic patients did not experience any exacerbation or change in their quality of life during their ACI. Secondly, ACI did not induce AHR in nonasthmatic subjects.

Viral infections are a major cause of asthma exacerbations in infants and in adults and were shown to be associated with approximately $80 \%$ of acute asthma exacerbations in children [3]. The rhinovirus is the most common agent implicated in asthma exacerbation. Others viruses implicated are respiratory syncitial viruses (RSV) and parainfluenza viruses, adenoviruses, enteroviruses and coronaviruses. Influenza virus is only found during annual epidemics. Experimental rhinovirus infection in asthmatic patients induces lower respiratory tract symptoms typical of mild asthma exacerbation [4]. Respiratory viruses enter and replicate in epithelial cells lining the upper as well as the lower airways. Virus-induced epithelial damage causes narrowing through dead epithelial cells dropping into the lumen, decreased mucociliary clearance, increased exposure of sensory nerves to irritants and decreased production of bronchodilating (prostaglandin $\mathrm{E}_{2}, \mathrm{NO}$, endopeptidase) and/ or decreased metabolism of bronchoconstricting (substance $\mathrm{P}$, neurokinin A) substances [5]. Moreover, virus-induced AHR is greater and longer in respiratory allergic patients than in control subjects, resulting in prolongation of variable clinical symptoms [6].

An interesting study was conducted two decades ago in tracheal explants to determine the susceptibility of these cultures to infection with nine mainly nonrespiratory viruses including chikungunya [7]. They found that tracheal cultures did not support multiplication of Japanese encephalitis and chikungunya viruses, as others viruses (herpes simplex 1 and 2, chandipura, West Nile, sandfly fever and polio-1) multiplied. Most of the viruses inducing asthma exacerbation are aerocontaminant ones. Their primary target is the respiratory epithelial cell. Conversely, chikungunya is an inoculated virus. In the study by BHONDE et al. [7], the viruses have a different cycle compared with aerocontaminant viruses but may secondarily proliferate in the tracheal cells. These ex vivo results showing the absence of tracheal proliferation of the chikungunya virus are in accordance with our clinical findings. ACI did not induce exacerbation in asthmatics patients as indicated by the absence of worsening in PFT. The mini-AQLQ score is a tool that evaluates the impact of asthma on quality of life. This issue was of major importance for patients with chikungunya who complained of major disability. The three AQLQ domains related to asthma symptoms and environmental exposure were not influenced by ACI. However, there was a trend in the domain analysing the physical activities. The mini-AQLQ is a self-administered questionnaire focusing on asthma. Obviously, it was difficult for patients to differentiate asthma-induced limitations with fatigue and joint pain related to ACI. However, the overall score was not statistically significant before and during ACI, clearly indicating that chikungunya infection did not induce exacerbation for asthmatic patients. The level of maintenance and rescue therapy was also not modified. AHR is a common feature in asthma, but viral infection also induces nonspecific AHR in healthy subjects that generally lasts $6-8$ weeks. 10 out of 11 nonasthmatic subjects had a negative metacholine challenge test. These results may suggest that ACI does not induce bronchial damage resulting in AHR. Only one subject had a positive metacholine provocation test and the test remains positive 6 months later. A thorough inquiry revealed the existence of asthma in the subject's siblings and a personal history of transient wheezing. This subject was secondarily considered as intermittent asthmatic.

Little is known on the pathophysiology of chikungunya infection. A recent report from south India indicated that chikungunya-affected patients were more frequently coinfected by respiratory viruses than chikungunya disease-free subjects [8]. However, no clinical data were described in this study, and chikungunya was not reported to induce any respiratory symptoms. Clinical reports demonstrated that the chikungunya virus involved target organs. Joints and skin are systematically injured [9]. Less frequent but of high clinical implication, chikungunya virus affected the liver and the brain, leading to severe hepatitis and encephalopathy. All in vitro or animal studies focused on joints, skin, brain, liver injury, but the lungs or bronchi were never studied. Chikungunya virus was found in human skeletal muscle, where it proliferates [10]. However, bronchial reactivity depends on smooth muscle constriction and, to date, no data are available to assess the presence of chikungunya virus in the airway musculature.

Our study has several limitations as we investigated an indirect effect of the viral infection. Unfortunately, we did not identify and quantify the virus itself in the sputum or in the nose by lavage or brushing. However, RT-PCR reflects the viral load and remains on average positive during 1 week [2]. During this acute phase, the viral load was extremely high, ranging from $10^{4}$ to $10^{9}$ copies $\cdot \mathrm{mL}^{-1}$ ( $10^{6}$ on average). Another limitation concerns the variability of AHR in asthmatic patients. The metacholine challenge test is more specific for the monitoring of AHR and allows the variation of AHR in asthmatics to be quantified. It may be possible that our asthmatic patients experienced a slight increase in their AHR that was not measurable by PFT and subjective clinical symptoms. Our asthmatic patients did not routinely have a baseline metacholine challenge test, so it was impossible to ascertain that AHR did not vary during ACI (table 1).

We conclude that chikungunya virus acute infection did not induce asthma exacerbations in asthmatic patients and did not induce AHR in infected nonasthmatics. The bronchi are not a target for ACI, but more data are needed to assess whether the entire lung is involved by chikungunya virus infection or not.

\section{F. Paganin*, C. Tasset*, P. Poubeau\#, V. Cochet ${ }^{\oplus}$ and G. Borgherini"}

*Service de Pneumologie and "Service des Maladies Infectieuses, GHSR, St Pierre, Réunion, and `St Gilles les Hauts, Réunion, France. 
Correspondence: F. Paganin, Service de Pneumologie et Maladies Infectieuses, GHSR, BP350, 97410 St Pierre, Réunion, France. E-mail: f.paganin@orange.fr

Statement of Interest: None declared.

Acknowledgements: The authors would like to thank F. Lilienthal, Philadelphia (PA, USA) for helpful advice and the review of the manuscript and P. Chanez (Service de Pneumologie, Université de Marseille, France) for his kind help.

\section{REFERENCES}

1 Renault P, Solet JL, Sissoko D, et al. A major epidemic of chikungunya virus infection on Reunion Island, France, 20052006. Am J Trop Med Hyg 2007; 77: 727-731.

2 Laurent P, Le Roux K, Grivard P, et al. Development of a sensitive real-time reverse transcriptase PCR assay with an internal control to detect and quantify chikungunya virus. Clin Chem 2007; 53: 1408-1414.

3 Johnston SL, Pattemore PK, Sanderson G, et al. The relationship between upper respiratory infections and hospital admissions for asthma: a time-trend analysis. Am J Respir Crit Care Med 1996; 154 654-660.

4 Gern JE, Vrtis R, Grindle KA, et al. Relationship of upper and lower airway cytokines to outcome of experimental rhinovirus infection. Am J Respir Crit Care Med 2000; 162: 2226-2231.

5 Papadopoulos NG, Xepapadaki P, Mallia P, et al. Mechanisms of virus-induced asthma exacerbations: state-of-the-art. A GA2LEN and InterAirways document. Allergy 2007; 62: 457-470.

6 Folkerts G, Busse WW, Nijkamp FP, et al. Virus-induced airway hyperresponsiveness and asthma. Am J Respir Crit Care Med 1998; 157: 1708-1720.

7 Bhonde RR, Wagh UV, Gupta NP. Replication of non-respiratory viruses in tracheal organ cultures. Br J Exp Pathol 1983; 64: 1-5.

8 Sankari T, Hoti SL, Govindaraj V, et al. Chikungunya and respiratory viral infections. Lancet Infect Dis 2008; 8: 3-4.

9 Parola P, Simon F, Oliver M. Tenosynovitis and vascular disorders associated with Chikungunya virus-related rheumatism. Clin Infect Dis 2007; 45: 801-802.

10 Ozden S, Huerre M, Riviere JP, et al. Human muscle satellite cells as targets of Chikungunya virus infection. PLoS ONE 2007; 2: e527.

DOI: $10.1183 / 09031936.00172909$

\section{Reversal of periodic breathing after aerobic training in heart failure}

\section{To the Editors:}

A 58-yr-old male was referred for participation in a cardiac rehabilitation programme. He had a long-term history of hypertensive cardiomyopathy and systolic dysfunction and was clinically stable, being treated with $\beta$-blocker carvedilol, losartan, digitalis, diuretics and spironolactone, with no changes in medication in the previous 16 weeks. An echocardiogram showed a $17 \%$ left ventricular ejection fraction and the patient was in New York Heart Association Functional Class III.

Before starting the exercise training programme, the patient was submitted to a maximal progressive cardiopulmonary exercise test, on an electromagnetically braked cycle ergometer (Medifit 400L; Medical Fitness Equipment, Maarn, The Netherlands), with work rate increments of $5 \mathrm{~W}$ every $1 \mathrm{~min}$ at $60 \mathrm{rpm}$ until exhaustion. Oxygen uptake $\left(V^{\prime} \mathrm{O}_{2}\right)$ and carbon dioxide production were determined by means of gas exchange on a breath-bybreath basis in a computerised system (Vmax 229 model; SensorMedics, BuenaVista, CA, USA). Peak $V^{\prime} \mathrm{O}_{2}$ was defined as the maximum attained $V^{\prime} \mathrm{O}_{2}$ at the end of the exercise period in which the patient could no longer maintain the cycle rate. During this test, the patient showed a very low exercise capacity, with a peak $V^{\prime} \mathrm{O}_{2}$ of $9.3 \mathrm{~mL} \cdot \mathrm{kg}^{-1} \cdot \mathrm{min}^{-1}$ and $35 \mathrm{~W}$ of peak power load. Evaluation of ventilation during exercise revealed the presence of periodic breathing (fig. 1), as identified by the criteria proposed by LeITE et al. [1] and others [2].

The patient underwent 4 months of exercise training, which consisted of $60-\mathrm{min}$ exercise sessions 3 times $^{\cdot}$ week $^{-1}$ under medical supervision at the Heart Institute (affiliation) [3]. Each exercise session consisted of 5 min stretching exercises, 25 min cycling on an cycle ergometer in the first month and up to $40 \mathrm{~min}$ in the next 3 months, $10 \mathrm{~min}$ local strengthening exercises (sit-ups, push-ups and pull-ups) and $5 \mathrm{~min}$ of cool down with stretching exercises. The exercise intensity was established by heart-rate levels that corresponded to anaerobic threshold up to $10 \%$ below the respiratory compensation point obtained in the cardiopulmonary exercise test. When a training

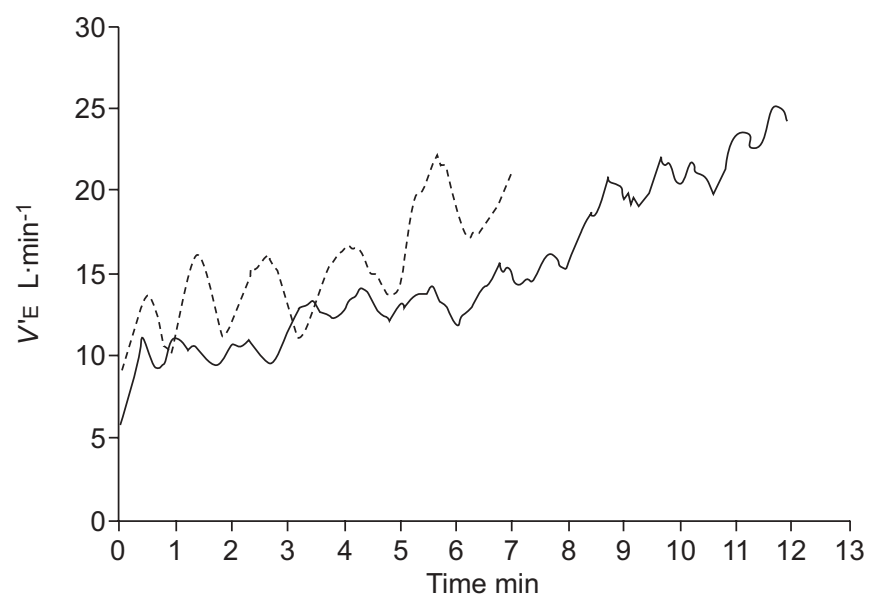

FIGURE 1. Ventilatory responses to graded cycle exercise of a patient with heart failure before (----) and after (-) four months of exercise training. V'E: minute ventilation. 